\title{
Crosslink in bone collagen in Paget's disease
}

\author{
D. P. MISRA
}

From Good Hope Hospital, Sutton Coldfield, West Midlands

SYNOPSIS The crosslink in bone collagen was analysed in specimens of bone obtained at necropsy from cases of Paget's disease and compared with normal bone collagen of the same age. The specimens were stored at $-20^{\circ} \mathrm{C}$ before analysis.

The predominant crosslink in a normal bone collagen was hydroxylysinohydroxynorleucine (di OH-LNL) (F1), which was designated syndesine in the past; another fraction, hydroxylysinorleucine (HLNL) (F2), much less prominent than di OH-LNL, was also noted in a normal bone collagen. Both fractions were reduced in bone tissue of advancing age. The peak corresponding to HLNL was considerably increased in Paget's disease. This abnormality was constantly seen in specimens of bone from cases of Paget's disease, but the significance of the finding could not be assessed from the present investigation.

Calcitonin has been shown to produce complete remission in Paget's disease and the crosslink pattern was found to be normal in specimens examined from a calcitonin-treated patient. This shows that calcitonin has some effect on the metabolism of collagen and a normal crosslink in such a situation lends support to this idea.

The study of crosslinks in collagen has added much to our knowledge of collagen chemistry but its application to clinical problems requires further investigation. It is generally accepted that the collagen fibres are stabilized by a system of covalent crosslinks (Bailey, 1968; Veis, 1967), and their formation is linked intimately with growth and development. Reduction with borohydride and subsequent analysis of acid hydrolysate of various collagen tissues showed that the more soluble collagen contained labile aldimine bonds (F2), whereas di OH-LNL (F1) was the only significant reducible crosslink in bone collagen (Bailey, 1970). Further investigation confirmed the identity of fraction 2 as HLNL and it was suggested that the crosslink existed in the fibre in the aldimine bond (Schiff base) as dehydrohydroxylysinonorleucine (Fowler and Bailey, 1972). It was also shown that HLNL accounted for $33 \%$ of the tritium activity in the column effluent from a hydrolysate of normal bone collagen treated with ${ }^{3} \mathrm{H}$ borohydride (Eyre and Glimcher, 1972).

Paget's disease is associated with increased turnover of bone. There is resorption and resynthesis of bone matrix. The serum acid and alkaline

Received for publication 27 November 1974. phosphatase is raised and hydroxyproline excretion is increased. The defect in Paget's disease causes a disturbance in bone metabolism and for this reason it was decided to analyse the crosslink in bone of Paget's disease and compare it with normal bone of the same age. It was also intended to analyse specimens of affected bone from patients with Paget's disease who had been treated with calcitonin.

\section{Materials and Method}

Specimens of bone from skull and pelvis were obtained at necropsy from three groups of patients between the ages of 60 and 75: (1) 15 specimens of normal bone from 10 necropsies (six men and four women) and five specimens of normal bone from three patients aged 15-20 years who died in road traffic accidents; (2) 10 specimens of bone affected with Paget's disease from eight patients (three men and five women); (3) on one occasion three specimens of bone were obtained from a 60-year-old man with advanced Paget's disease who had been treated with calcitonin for two months before his death. The specimens were collected from the affected bones located during life by bone radiography.

Soft tissue was dissected from the bone, which was then crushed and decalcified with 0.35 M EDTA 
(pH 8.9). The tissue was suspended in $0.9 \% \mathrm{NaCl}$

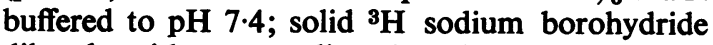
diluted with non-radioactive borohydride was added directly to the suspension of bone tissue and $\mathrm{NaCl}$, which was stirred every 20 minutes. Six mg of non-radioactive sodium borohydride was mixed with $25 \mathrm{mCi}$ of ${ }^{3} \mathrm{H}$ borohydride obtained from the Radiochemical Centre, Amersham, and used for 25 specimens. The reduction was allowed to proceed for two hours at room temperature, stopped by the addition of acetic acid to lower the $\mathrm{pH}$ to 4 , and the suspension dialysed against distilled water for 24 hours. The tissue was then freeze-dried and hydrolysed.

Two hundred $\mathrm{mg}$ of the reduced, freeze-dried collagen was hydrolysed in about $25 \mathrm{ml}$ of constant boiling $6 \mathrm{n} \mathrm{HCl}$ for 24 hours. The $\mathrm{HCl}$ was removed by rotary evaporation, the sample then being taken up in distilled water, this too being removed by rotary evaporation. The sample was finally dissolved in about $5 \mathrm{ml}$ of pyridine/formic acid starting buffer pH 2.90 and applied to the column. The column was filled with Zeo-carb 225 and maintained at $60^{\circ} \mathrm{C}$. The amino acids were eluted with a gradient formed by running 1.0 M-pyridine-formic acid buffer, pH 2.9. A split-stream device was attached to the end of the column and one-twentieth of the eluate pumped through the modified ninhydrin system, water being used to make up the volume. The remainder of the eluate was collected with an automatic fraction collector. The flow rate was adjusted to $40 \mathrm{ml} / \mathrm{h}$ and $10-\mathrm{min}$ fractions were collected. The column was regenerated with 2 M-pyridine and then starting buffer.

About 100 fractions were collected and analysed for radioactivity by dissolving a portion $(0.2 \mathrm{ml})$ in $3 \mathrm{ml}$ of Bray's solution and measuring the ${ }^{3} \mathrm{H}$ radioactivity with a Packard 3375 liquid scintillation counter. Amino acids were identified by chromatography against standard amino acids with the singlephase system butan-1-ol-acetic acid-water $(4: 1: 1$ by volume). The details of the method have been described by Bailey, Peach, and Fowler (1970).

\section{Results}

Reduction of normal bone collagen with ${ }^{3} \mathrm{H}$ borohydride and subsequent hydrolysis and analysis of the radioactive peaks of the column fraction showed a prominent peak, di OH-LNL (F1).

A second fraction which corresponds to HLNL (F2) but much less prominent than F1 was also noted in normal bone collagen (fig 1).

The proportion of fractions (F1 and F2) varied between 6:1 and 2:1 in normal cases. Both fractions were reduced in specimens of bone obtained from

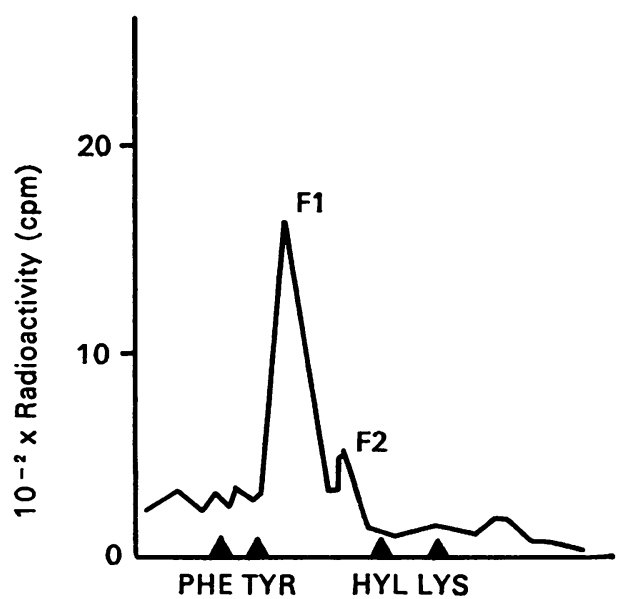

Fig 1 Normal skull bone of patient aged 70. Distribution of ${ }^{3} \mathrm{H}$ radioactivity of acid hydrolysates of reduced bone collagen. Hatched areas, denote position of normal amino acids; $F 1$ denotes di $O H-L N L$ peak and $F 2$ corresponds to $H L N L$.

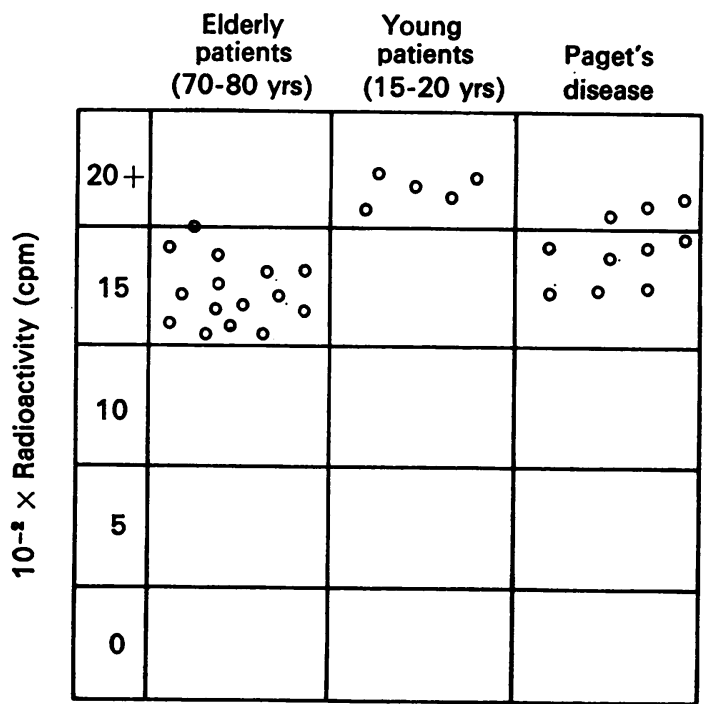

Fig. 2 Peak $\mathrm{H}^{3}$ activity in the region of di $O H-L N L$ in normal young and old subjects and patients with Paget's disease.

elderly people when compared with those from young people (fig 2). This ratio was altered in Paget's disease, which was due to an increase in HLNL (fig 3). The peak activity in the region of HLNL in normal and Paget's disease is shown in figure 4. Three specimens of bone examined from different affected sites in a patient treated with calcitonin shows a normal crosslink pattern. 


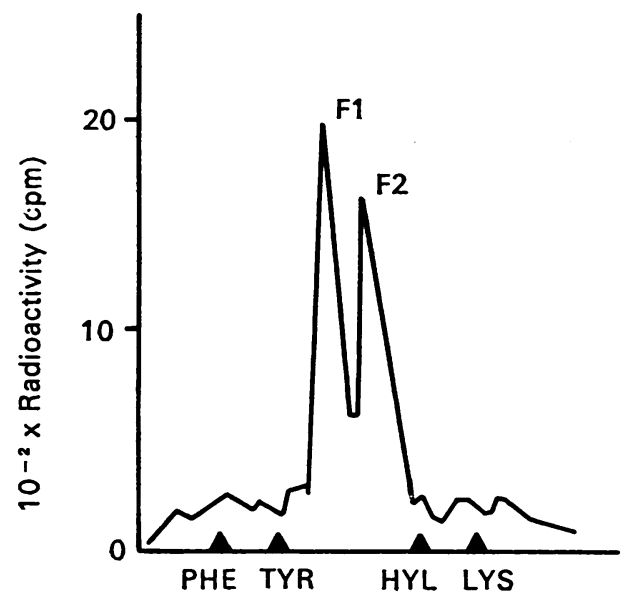

Fig 3 Paget's disease (skull bone) of patient aged 73. Distribution of ${ }^{3} \mathrm{H}$ radioactivity of acid hydrolysates of reduced bone collagen. Hatched areas denote position of normal amino acids; F1 denotes di $O H-L N L$ peak and F2 corresponds to $H L N L$.

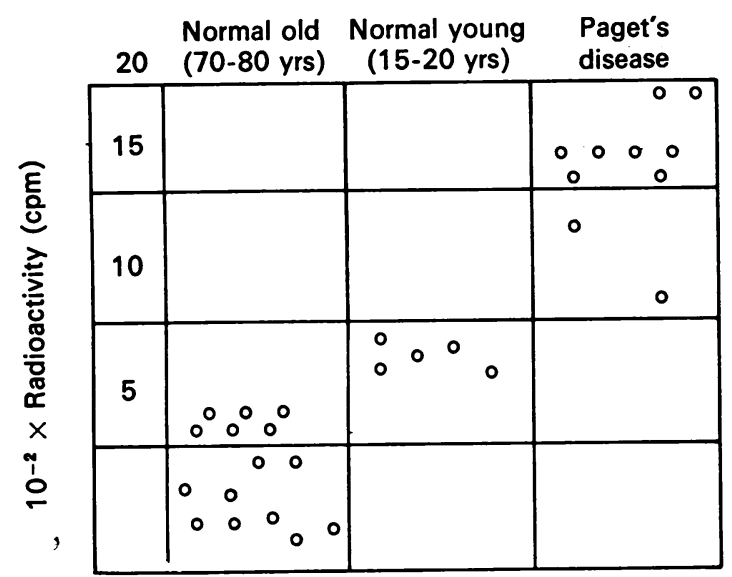

Fig. 4 Comparison of peak ${ }^{3} \mathrm{H}$ radioactivity in the region of HLNL in bone collagen in normal subjects and cases of Paget's disease.

\section{Discussion}

Collagen forms the major supporting framework of the body. Recent studies have shown that a protein monomer, tropocollagen, is the basic unit from which the collagen fibres are formed. This molecule consists of three polypeptide chains wound together in a helical form. There are strong covalent bonds crosslinking the tropocollagen molecules to stabilize the chains and form a three-dimensional network. The main crosslink in a bone collagen is di OH-LNL. This is a reduced aldimine derived from hydroxylysine (Mechanic, Gallop, and Tanzer, 1971; Davis and Bailey, 1971). In vivo it exists as hydroxylysino5-keto-norleucine (Bailey, Robins, and Balian, 1974).

A high level of HLNL was observed in bone collagen affected with Paget's disease, and HLNL forms the main crosslink component of a soluble collagen like skin. The biosynthetic pathways of HLNL and di OH-LNL have been shown to be different and consequently the interconversion of the two compounds does not take place. This suggests that the crosslink in bone collagen in Paget's disease is abnormal. Calcitonin has been shown to produce a beneficial effect in Paget's disease with complete remission of clinical, radiological, and biochemical changes (MacIntyre, Bayfield, Matthews, Moseley, and Woodhouse, 1972; Woodhouse, Bordier, Fisher, Joplin, Reiner, Kalu, Foster, and MacIntyre, 1971). The action of calcitonin is to prevent hypercalcaemia, which occurs during absorption of calcium from the diet, by its action on the bone. By this means the absorbed calcium is conserved for its use in the skeleton (Munson, Schwesinger, Cooper, Kenney Grey, and Tai-Chan Peng, 1972). Few specimens of affected bone located by radiography and analysed after death in a calcitonin-treated patient showed a normal fraction of HLNL. This suggests that calcitonin might have some action on collagen and its crosslink pattern, and it may be possible that the proper deposition of calcium is facilitated by this.

A decrease in the proportion of reducible crosslink (OH-LNL) was also observed in the bone collagen of elderly patients, which is in agreement with the findings of others (Robins, Shimokomaki, and Bailey, 1973). A high incidence of osteoporosis is also seen in the same age group. It was proposed that the stable bonds in the mature collagen were derived in some way from the reducible crosslinks (Bailey et al, 1974). It is also possible that in elderly subjects there is a reduction in the total mass of collagen in bone and consequently a decrease in the crosslinks.

I am grateful to the Departments of Biophysics and Biochemistry of the United Bristol Hospitals for their help in carrying out this work. My special thanks are due to Mr G. Staddon, United Bristol Hospitals, without whose help the work would not have been possible. I also wish to thank Dr D. D. Gibbs, Good Hope Hospital, who kindly looked at the paper and gave his suggestions and advice. 


\section{References}

Bailey, A. J. (1968). The nature of collagen. In Comprehensive Biochemistry, edited by M. Florkin and E. H. Stotz, Vol. 26, Part B, pp. 297-243. Elsevier, Amsterdam.

Bailey, A. J. (1970). Comparative study of the nature of the crosslinks in the collagen of various fish tissues. Biochim. biophys. Acta (Amst.), 221, 652-656.

Bailey, A. J., Peach, C. M., and Fowler, L. J. (1970). Chemistry of the collagen crosslinks: isolation and characterization of two intermediate intermolecular cross-links in collagen. Biochem. $J ., 117,819-831$.

Bailey, A. J., Robins, S. P., and Balian, G. (1974). Biological significance of the intermolecular crosslinks of collagen. Nature (Lond.), 251, 105-109.

Davis, N. R., and Bailey, A. J. (1971). Chemical synthesis of the reduced form of an intermolecular crosslink of collagen: a re-evaluation of the structure of syndesine. Biochem. biophys. Res. Commun., 45, 1416-1422.

Eyre, D. R., and Glimcher, M. J. (1972). Reducible crosslinks in hydroxylysine deficient collagens of a heritable disorder of connective tissue. Proc. nat. Acad. Sci. (Wash.), 69, 25942598.

Fowler, L. J., and Bailey, A. J. (1972). Current concepts of the crosslinking in bone collagen. Clin. Orthop. related Res. 85, 193-206.
MacIntyre, P. G. H., Bayfield, L. G., Matthews, E. W., Moseley, J. M., and Woodhouse, N. J. Y. (1972). Studies of the effects of human synthetic calcitonin in experimental animals and man. In Endocrinology 1971: Proceedings of the Third International Symposium, London, pp. 21-26. Heinemann, London.

Mechanic, G. L., Gallop, P. M., and Tanzer, M. L. (1971). The nature of crosslinking in collagens from mineralized tissues. Biochem. biophys. Res. Commun., 45, 644-653.

Munson, P. L., Schwesinger, W. H., Cooper, C. W., Kenney Grey, T., and Tai-Chan Peng (1972). Physiological function of thyrocalcitonin. In Endocrinology 1971: Proceedings of the Third International Symposium, London, pp. 98-107. Heinemann, London.

Robins, S. P., Shimokomaki, M., and Bailey, A. J. (1973). The chemistry of collagen crosslinks; age related changes in the reducible components of intact bovine collagen fibres. Biochem. J., 131, 771-780.

Veis, A. (1967). Intact collagen. In Treatise on Collagen, Vol. 1, edited by G. N. Ramachandra. Academic Press, New York and London. pp. 367-439.

Woodhouse, N. J. Y., Bordier, P., Fisher, M., Joplin, G. F., Reiner, M., Kalu, D. N., Foster, G. V., and MacIntyre, J. (1971). Human calcitonin in the treatment of Paget's bone disease. Lancet, 1. 1139-1143. 